\title{
Engineering the acyltransferase domain of epothilone polyketide synthase to alter the substrate specificity
}

Huimin Wang ${ }^{1}$, Junheng Liang ${ }^{1}$, Qianwen Yue ${ }^{1}$, Long Li ${ }^{2}$, Yan Shi ${ }^{1}$, Guosong Chen ${ }^{2}$, Yue-zhong Li Xiaoying Bian ${ }^{3}$, Youming Zhang ${ }^{3}$, Guoping Zhao ${ }^{1,4}$ and Xiaoming Ding ${ }^{1 *}$ (D)

\begin{abstract}
Background: Polyketide synthases (PKSs) include ketone synthase (KS), acyltransferase (AT) and acyl carrier protein (ACP) domains to catalyse the elongation of polyketide chains. Some PKSs also contain ketoreductase (KR), dehydratase (DH) and enoylreductase (ER) domains as modification domains. Insertion, deletion or substitution of the catalytic domains may lead to the production of novel polyketide derivatives or to the accumulation of desired products. Epothilones are 16-membered macrolides that have been used as anticancer drugs. The substrate promiscuity of the module 4 AT domain of the epothilone PKS (EPOAT4) results in production of epothilone mixtures; substitution of this domain may change the ratios of epothilones. In addition, there are two dormant domains in module 9 of the epothilone PKS. Removing these redundant domains to generate a simpler and more efficient assembly line is a desirable goal.
\end{abstract}

Results: The substitution of module 4 drastically diminished the activity of epothilone PKS. However, with careful design of the KS-AT linker and the post-AT linker, replacing EPOAT4 with EPOAT2, EPOAT6, EPOAT7 or EPOAT8 (specifically incorporating methylmalonyl-CoA (MMCOA)) significantly increased the ratio of epothilone $D(\mathbf{4})$ to epothilone $C$ (3) (the highest ratio of $\mathbf{4 : 3}=4.6: 1$ ), whereas the ratio of $\mathbf{4 : 3}$ in the parental strain Schlegelella brevitalea 104-1 was 1.4:1. We also obtained three strains by swapping EPOAT4 with EPOAT3, EPOAT5, or EPOAT9, which specifically incorporate malonyl-COA (MCOA). These strains produced only epothilone $C$, and the yield was increased by a factor of 1.8 compared to that of parental strain 104-1. Furthermore, mutations of five residues in the AT domain identified Ser310 as the critical factor for MMCOA recognition in EPOAT4. Then, the mutation of His308 to valine or tyrosine combined with the mutation of Phe310 to serine further altered the product ratios. At the same time, we successfully deleted the inactive module $9 \mathrm{DH}$ and ER domains and fused the $4 \mathrm{KR}$ domain with the KR domain through an $\sim 25$-residue linker to generate a productive and simplified epothilone PKS.

Conclusions: These results suggested that the substitution and deletion of catalytic domains effectively produces desirable compounds and that selection of the linkers between domains is crucial for maintaining intact PKS catalytic activity.

\footnotetext{
*Correspondence: xmding74@fudan.edu.cn

${ }^{1}$ Collaborative Innovation Center for Genetics and Development, State

Key Laboratory of Genetic Engineering, Shanghai Engineering Research Center of Industrial Microorganisms, Department of Microbiology, School

of Life Sciences, Fudan University, Shanghai 200438, People's Republic

of China

Full list of author information is available at the end of the article
}

(C) The Author(s) 2021. This article is licensed under a Creative Commons Attribution 4.0 International License, which permits use, sharing, adaptation, distribution and reproduction in any medium or format, as long as you give appropriate credit to the original author(s) and the source, provide a link to the Creative Commons licence, and indicate if changes were made. The images or other third party material in this article are included in the article's Creative Commons licence, unless indicated otherwise in a credit line to the material. If material is not included in the article's Creative Commons licence and your intended use is not permitted by statutory regulation or exceeds the permitted use, you will need to obtain permission directly from the copyright holder. To view a copy of this licence, visit http://creativeco mmons.org/licenses/by/4.0/. The Creative Commons Public Domain Dedication waiver (http://creativecommons.org/publicdomain/ zero/1.0/) applies to the data made available in this article, unless otherwise stated in a credit line to the data. 
Keywords: Polyketide synthase, AT, Epothilone, Domain swap, Substrate specificity

\section{Background}

Nonribosomal peptides (NRPs) and polyketides (PKs) are two prominent families of natural products, some of which exhibit high bioactivity [1, 2]. Nonribosomal peptide synthetases (NRPSs) and polyketide synthases (PKSs) assemble amino acids and simple acyl-CoA building blocks into the final complex compounds NRPs and PKs, respectively [3-5]. Each PKS module contains three necessary domains, the ketosynthase (KS) domain, acyltransferase (AT) domain, and acyl carrier protein (ACP) domain [6-8]. Some modules also contain optional processing domains, a ketoreductase ( $\Psi \mathrm{KR} / \mathrm{KR})$ domain, a dehydratase (DH) domain, and an enoylreductase (ER) domain that are used for successively catalysing the reduction of the resulting $\beta$-keto group $[9,10]$. Notably, among processing domains, $\Psi$ KR acts not as a catalytic domain but as a structural domain to support the integrity of the active site of the catalytic KR domain [11]. The thioesterase (TE) domain releases the eventual polyketide compounds. The collinearity (strict correlation of the PKS organization and the product structure) of PKSs has sparked interest in the generation of new derivatives by PKS engineering [12-16].

Epothilones are members of the NRP/PK family synthesized by hybrid NRPS/PKS synthetases, which stabilize microtubules in parallel with taxol and have been used as anticancer agents [17]. The natural producer myxobacterium Sorangium cellulosum produces epothilone $\mathrm{A}$ and $\mathrm{B}$, while epothilone $\mathrm{C}$ and $\mathrm{D}$ are produced by inactivation or deletion of epoK, which is an epoxidase gene in the epothilone biosynthesis gene cluster (MIBiG-ID BGC0000989) [18]. Epothilone B and $\mathrm{D}$ were proved to have higher activity than epothilone $A$ and $C$ [19]. The marketed anticancer drug ixabepilone $\left(\right.$ IXEMPRA $^{\mathrm{TM}}$ ) is a Food and Drug Administration (FDA)-approved synthetic analogue of epothilone B, and utidelone (epothilone D) could effectively treat breast cancer in phase 3 trials [20,21]. Mixtures of epothilones were generated due to the module 4 native AT domain of the epothilone PKS (EPOAT4), which can add either methylmalonyl-CoA (MMCoA) or malonyl-CoA (MCoA) extender units at positions $\mathrm{C} 11-\mathrm{C} 12$ during polyketide chain elongation [22]. We wanted to engineer EPOAT4 for two main reasons. First, the production of mixtures of compounds is a disadvantage in industrial production, and changing the ratios to favour preferred compounds has commercial potential. Second, the unique substrate specificity of EPOAT4 makes it an excellent model for the comparison of MMCoA and MCoA incorporation in vivo to improve our understanding of the AT domain. The AT engineering mainly include three strategies: swapping domains, exchanging modules, and site-directed mutagenesis of PKS genes. EPOAT4 has been used as a donor AT in the engineering of 6-dEB synthase (DEBS) $[22,23]$, but to date, EPOAT4 has not been engineered in epothilone PKS in vivo. The genetic manipulation in the original epothilone producer is difficult. In previous studies, epothilone PKS was modified in heterologous host Myxococcus xanthus by the inactivation of KR, DH or ER domains in EpoD, resulted in production of novel epothilone derivatives but in lower yields for use in industrial production [18, 24, 25]. Moreover, engineering AT domains resulted in novel polyketides but usually lower yields in early studies due to a lack of understanding of the structure of AT [26-29].

Moreover, module 9 of the epothilone PKS (EPOM9) is a sufficiently reducing module with a complete modifying region, and we observed that the $\mathrm{DH}$ and ER domains of module 9 (EPODH9 and EPOER9) are inactive (Fig. 1). Inactive domains are common in PKSs but often ignored; for example, there are five dormant domains in rapamycin PKS [30] and six inactive domains in rifamycin PKS [31]. We speculated that the deletion of these redundant domains could reduce the size of the epothilone gene cluster and might simultaneously improve the efficiency of epothilone PKS. We also used EPOM9 as a model to explore whether the inactive processing domains could be removed freely, providing an engineering strategy for other PKSs. Unlike the partly reducing module (KS-ATYKR-KR-ACP), the catalytic KR domain and non-catalytic $\Psi K R$ are split by the ER domain in fully reducing modules (KS-AT-DH-YKR-ER-KR-ACP). Structural information shows that the $\Psi \mathrm{KR} / \mathrm{KR}$ domains are laterally tethered, that there is an absence of stable interfaces between the different reductive domains, and that the KR domain does not contact its adjacent domains [32] (Additional file 1: Figure S1a). These facts suggest the possibility of deleting inactive EPODH9 and EPOER9 without affecting the activity of epothilone PKS.

In our previous work, we successfully expressed epothilone PKS in the heterologous host Schlegelella brevitalea DSM 7029 and obtained mutant strains that produced high levels of epothilone $\mathrm{C}$ and $\mathrm{D}$ by the deletion of epoK [33]. The high epothilone yields and facile genetic manipulation in our heterologous host provide us a platform to engineer epothilone PKS. Herein, we rationally engineered domains in module 4 of the epothilone PKS (EPOM4) and EPOM9 and constructed a series of 


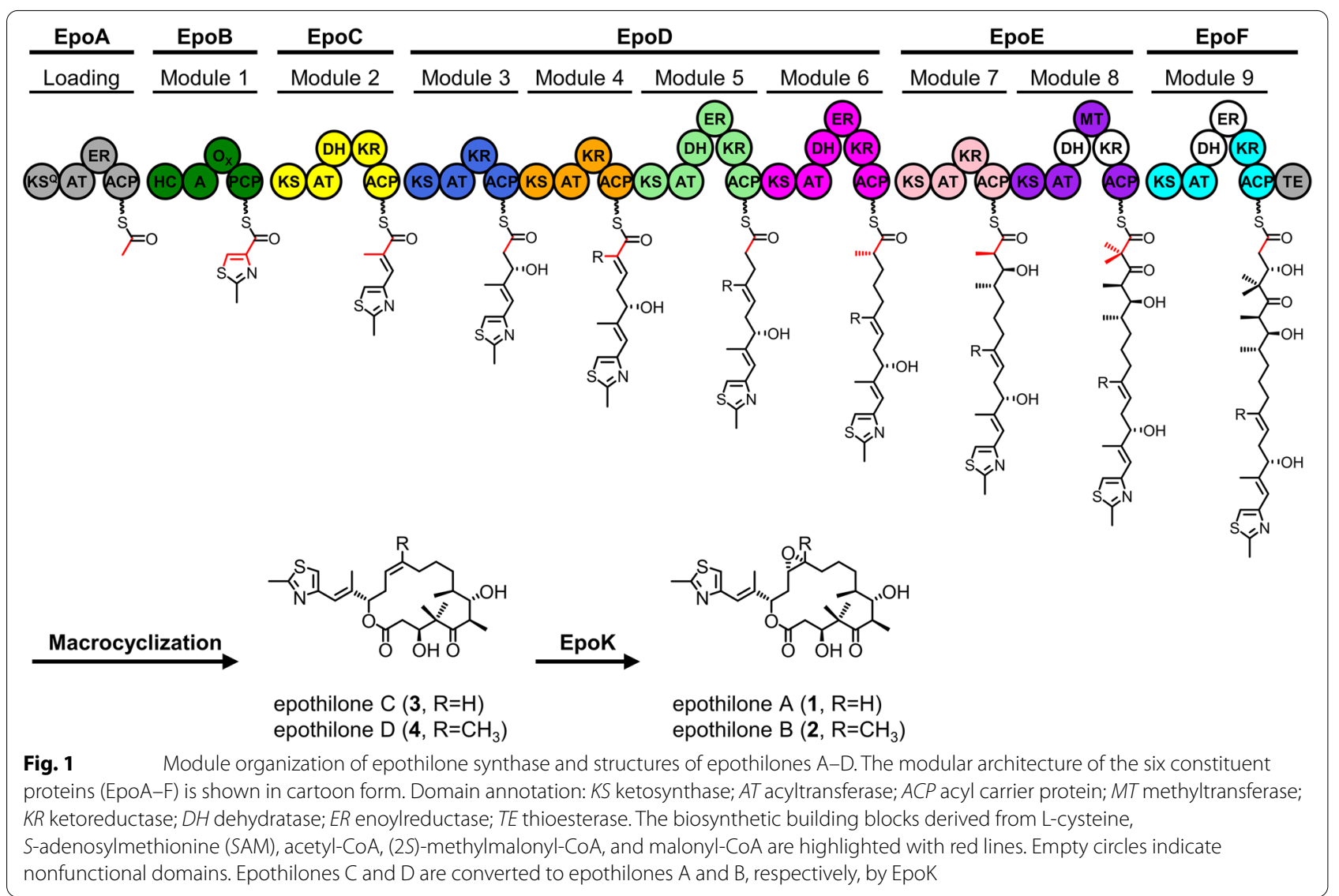

hybrid epothilone PKSs in the heterologous epothilone producer S. brevitalea. We significantly increased the proportion of epothilone D and obtained three strains that produced only epothilone C. We also successfully deleted the inactive DH and ER domains in EPOM9 and fused $\Psi K R$ with KR through an $\sim 25$-residue linker to generate a productive epothilone PKS and simplified the completely reducing region to a partly reducing region in EPOM9 without a decrease in the production level. Finally, our study further explored the importance and the sequence flexibility of $\Psi$ KR-KR-linker.

\section{Results}

The turnover rates of chimaeric epothilone PKSs designed by module 4 exchanges were drastically diminished

Engineering the AT specificity can alter the carbon skeleton of the polyketide by selecting different acylCoA substrates. To compare three strategies in altering AT specificity, we first exchanged the module in which EPOAT4 is located. The previously optimized highyield epothilone producer $S$. brevitalea 104-1 produced $27.7 \mathrm{mg} / \mathrm{l}$ epothilone D (4) and $19.9 \mathrm{mg} / \mathrm{l}$ epothilone C (3) $(4: 3=1.4: 1)$ as a consequence of EPOAT4 incorporating either MMCoA or MCoA extender units during polyketide chain elongation [34]. We exchanged EPOM4 with modules that harbour MMCoA-specific AT domains. Four chimaeric epothilone PKSs were constructed in which EPOM4 was replaced by module 7 of the epothilone PKS (EPOM7), module 6 from the erythromycin PKS (ERYM6), and module 4 or 10 from the rapamycin PKS (RAPM4 and RAPM10) (Additional file 1: Figure S2). To preserve interactions between modules related to the catalytic integrity of the epothilone PKS, EPOM4 was replaced by EPOM7 in a manner that retained the natural intermodular linker between EPOM3 and EPOM4 and between EPOM7 and EPOM8 to generate MMR2044 (EPOM7) (Additional file 1: Table S1). No downstream modules were connected to the modules for the other three fusions, MMR2024 (ERYM6), MMR2027 (RAPM4), and MMR2026 (RAPM10), through covalent linkers in their natural polypeptides. Thus, in these three mutants, EPOM3, the heterologous modules and EPOM5 were joined by two covalent linkers from EpoD (Additional file 1: Table S1). However, the production of epothilones in MMR2027 and MMR2026 was completely abolished. MMR2024 and MMR2044 yielded detectable amounts of epothilone $\mathrm{C}$ and $\mathrm{D}$, as shown by LC-MS (Additional file 1: Figure S3), but the yields were 
quite low. MMR2024 produced only $4 \mu \mathrm{g} / \mathrm{l}$ epothilone $\mathrm{C}$ and $14 \mu \mathrm{g} / \mathrm{l}$ epothilone D. MMR2044 produced $15 \mu \mathrm{g} / \mathrm{l}$ epothilone $\mathrm{C}$ and $17 \mu \mathrm{g} / \mathrm{l}$ epothilone $\mathrm{D}$ (Additional file 1 : Table S2).

\section{EPOAT4 domain swapping alters the ratio of epothilone D to epothilone $C$}

In engineering the EPOAT4 domain, both replacement of the entire module and domain swapping are possible. Considering that all chimaeric epothilone PKSs obtained through module exchange have lower product yields, we performed domain-swap experiments. We engineered EPOAT4 using a fruitful AT-swap strategy reported in 2016 by Yuzawa et al. [23]. We replaced EPOAT4 with several non-native AT domains that specifically incorporate MMCoA, including AT2, AT6, AT7, and AT8 of the epothilone PKS (EPOAT2, EPOAT6, EPOAT7, and EPOAT8), AT1 from the rapamycin PKS (RAPAT1), and AT6 of the erythromycin PKS (ERYAT6) (Fig. 2a). Nonnatural hybrid EPOM4 was generated by using optimal fusion sites located in the KS-AT linker (KAL) and the non-conserved $\mathrm{N}$-terminal region in the post-AT linker (PAL1) for AT domain exchanges; the highly conserved post-AT linker (PAL2) retained the original structure of EPOM4 (Additional file 1: Figure S4). It is believed that the interaction between the conserved PAL2 and the KS domain can stabilize the correct conformation of KS, which is essential for the catalysed chain extension reaction. The resulting four strains show different metabolic profiles from the parental strain 104-1. As shown in Fig. 2b, the epothilone D yields in MMR2048 (EPOAT2), MMR2049 (EPOAT6), MMR2017 (EPOAT7), and MMR2016 (EPOAT8) were $26.9 \mathrm{mg} / \mathrm{l}, 30.0 \mathrm{mg} / \mathrm{l}$, $28.3 \mathrm{mg} / \mathrm{l}$, and $29.7 \mathrm{mg} / \mathrm{l}$, respectively, as determined by HPLC analysis (Fig. 2c; Additional file 1: Figure S5). To our surprise, these four mutants still produced epothilone C. One possible explanation is that these four EPOAT domains could incorporate $\mathrm{MCoA}$ in their natural context, since a previous study showed that the natural producer myxobacterium $S$. cellulosum produced minor congeners harbouring $\mathrm{MCoA}$ at $\mathrm{C} 16, \mathrm{C} 8, \mathrm{C} 6$, or $\mathrm{C} 4$ of the epothilone structure [35]. Then, we compared the incorporation of two alternative substrates by EPOAT2, EPOAT6, EPOAT7 and EPOAT8, and the results showed that the ratio of epothilone $D$ to epothilone $C$ was significantly increased to approximately $3.2: 1,4.5: 1,4.6: 1$ and 3.9:1, i.e., enhanced by 1.3-, 2.2-, 2.3-, and 1.8-fold, respectively. However, the production of epothilones MMR2018 (RAPAT1) and MMR2012 (ERYAT6) were at lower yields (Additional file 1: Table S2, Figure S6). MMR2018 produced less than $1 \mu \mathrm{g} / \mathrm{l}$ epothilone $\mathrm{C}$ and $17 \mu \mathrm{g} / \mathrm{l}$ epothilone D. MMR2012 produced trace amounts of epothilone $\mathrm{C}$ and D (less than $1 \mu \mathrm{g} / \mathrm{l}$ ).
Subsequently, we replaced EPOAT4 of the epothilone PKS with three MCoA-specific AT domains derived from the same PKS, including EPOAT3, EPOAT5, and EPOAT9 (Fig. 3a). We used the same AT-swap strategy as above. Compared with the parental strain 104-1, all three mutant strains produced the desired epothilone $C$, while epothilone D disappeared (Fig. 3b, c; Additional file 1: Figure S5), which was consistent with the design expectations. The epothilone C yields in MMR2029 (EPOAT3), MMR2021 (EPOAT5), and MMR2020 (EPOAT9) were $55.1 \mathrm{mg} / \mathrm{l}, 42.5 \mathrm{mg} / \mathrm{l}$, and $47.3 \mathrm{mg} / \mathrm{l}$, respectively (Additional file 1: Table S2). Compared to those of the parental strain 104-1, the titres of epothilone $C$ increased by approximately 1.8-, 1.1- and 1.4-fold, respectively.

\section{Site-directed mutagenesis confirms that Ser 310 in the HASH motif is the key residue for MMCoA recognition by EPOAT4}

We conducted site-directed mutagenesis of the AT domain to investigate which residues are key to changing the substrate selectivity of this domain. The above result indicated that the AT-swap strain MMR2029 (EPOAT3) produced only epothilone C (Fig. 3b, c), illustrating the exclusive substrate specificity of EPOAT3 for MCoA. Based on amino acid sequence alignments of EPOAT3 (selecting MCoA) and EPOAT4 (selecting MMCoA or MCoA), we found that these two ATs differ in only nine amino acids but possess different substrate specificities (Fig. 4). Therefore, we speculated that some of these nine amino acids in EPOAT4 are correlated with MMCoA selectivity. After further sequence alignments of these nine amino acids in ten AT domains, we excluded four amino acids in EPOAT4: Pro165, Gln175, Val204, and Ala205. Pro165 and $\operatorname{Gln} 175$ are identical in three domains (EPOAT4, EPOAT5 and EPOAT9), while Val204 and Ala205 are identical in EPOAT4 and EPOAT9 (Additional file 1: Table S3). The above result also showed that EPOAT5 and EPOAT9 exclusively recognize MCoA (Fig. 3b, c), so we inferred that these four amino acids are not the reason for the EPOAT4 domain recognition of MMCoA. Then, we identified the remaining five residues (Thr185, Ala209, Ser310, Leu383, and Arg426) in the EPOAT4 domain. We individually mutated five residues from the MCoA-specific EPOAT3 domain in module 4 of the MMR2029 mutant to the corresponding residues of the MMCoA-specific EPOAT4 domain to give MMR2033 (A185T), MMR2034 (I209A), MMR2035 (F310S), MMR2039 (V383L), and MMR2040 (G426R). Compared to MMR2029 (which produced only epothilone $\mathrm{C}$ ), in these five variants, only mutation F310S resulted in a promiscuous AT domain that incorporated both extender units, and the proportion 


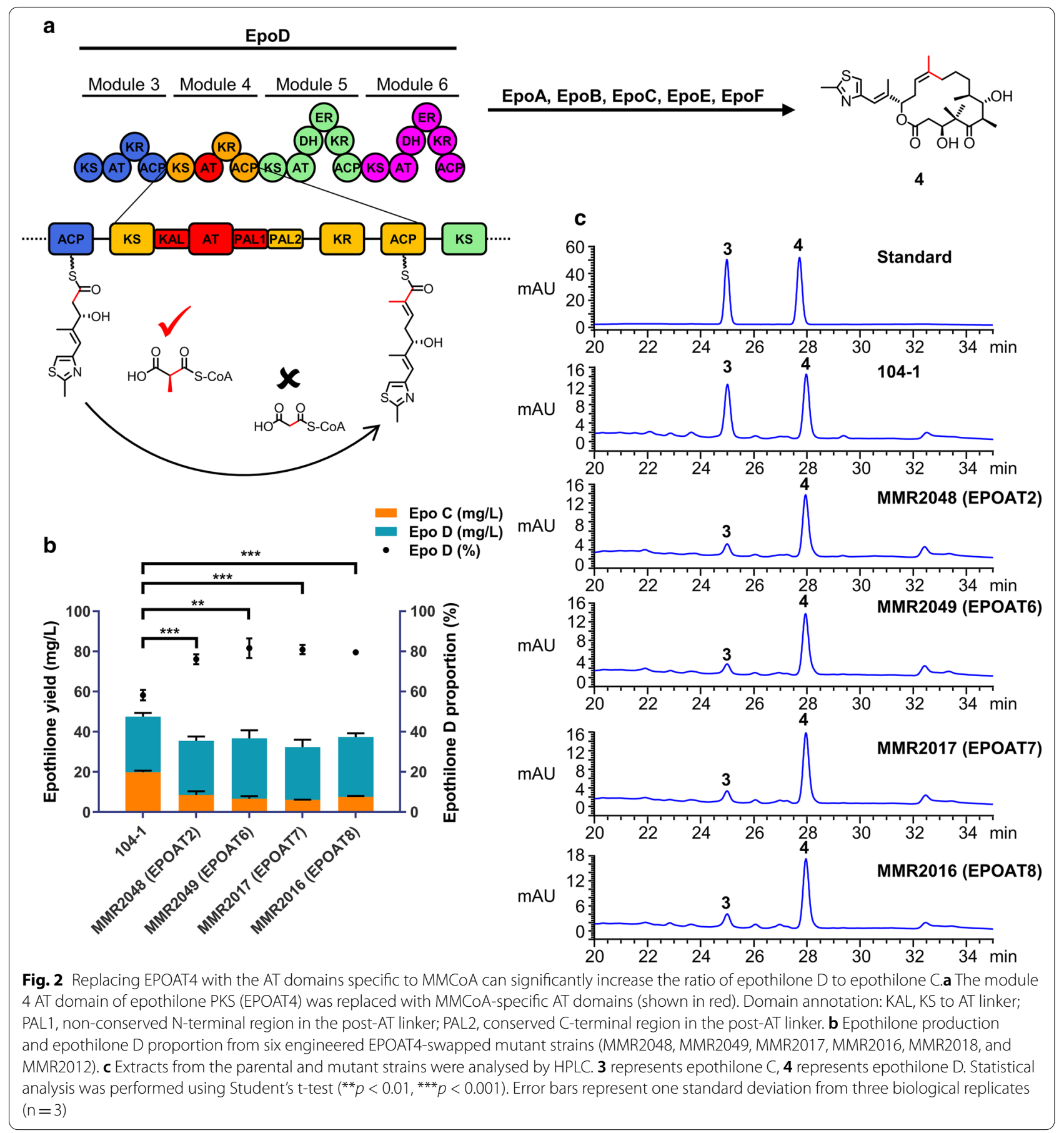

of epothilone D varied from 0 to $36 \%$ (Fig. 5a, b; Additional file 1: Table S2, Figure S7). These results indicate that among the residues that are different in EPOAT3 and EPOAT4, Ser310 is responsible for the MMCoA selectivity of EPOAT4. To verify this conclusion, we mutated Ser310 from the EPOAT4 of the parental strain 104-1 to phenylalanine and resulting MMR2055 (S310F). Expectedly, mutation S310F resulted in an exclusive substrate specificity of EPOAT4 for MCoA and MMR2055 (S310F) produced $50.3 \mathrm{mg} / \mathrm{l}$ epothilone C (Fig. 5a, b).

Phe310 and Ser310 are located in the HAFH motif and the YASH motif, respectively, and according to previous studies, they are related to MCoA and MMCoA specificity, respectively [36]. This motif shows divergent sequences in EPOAT3 (HAFH), ERYAT6/RAPAT1 (YASH), EPOAT7 


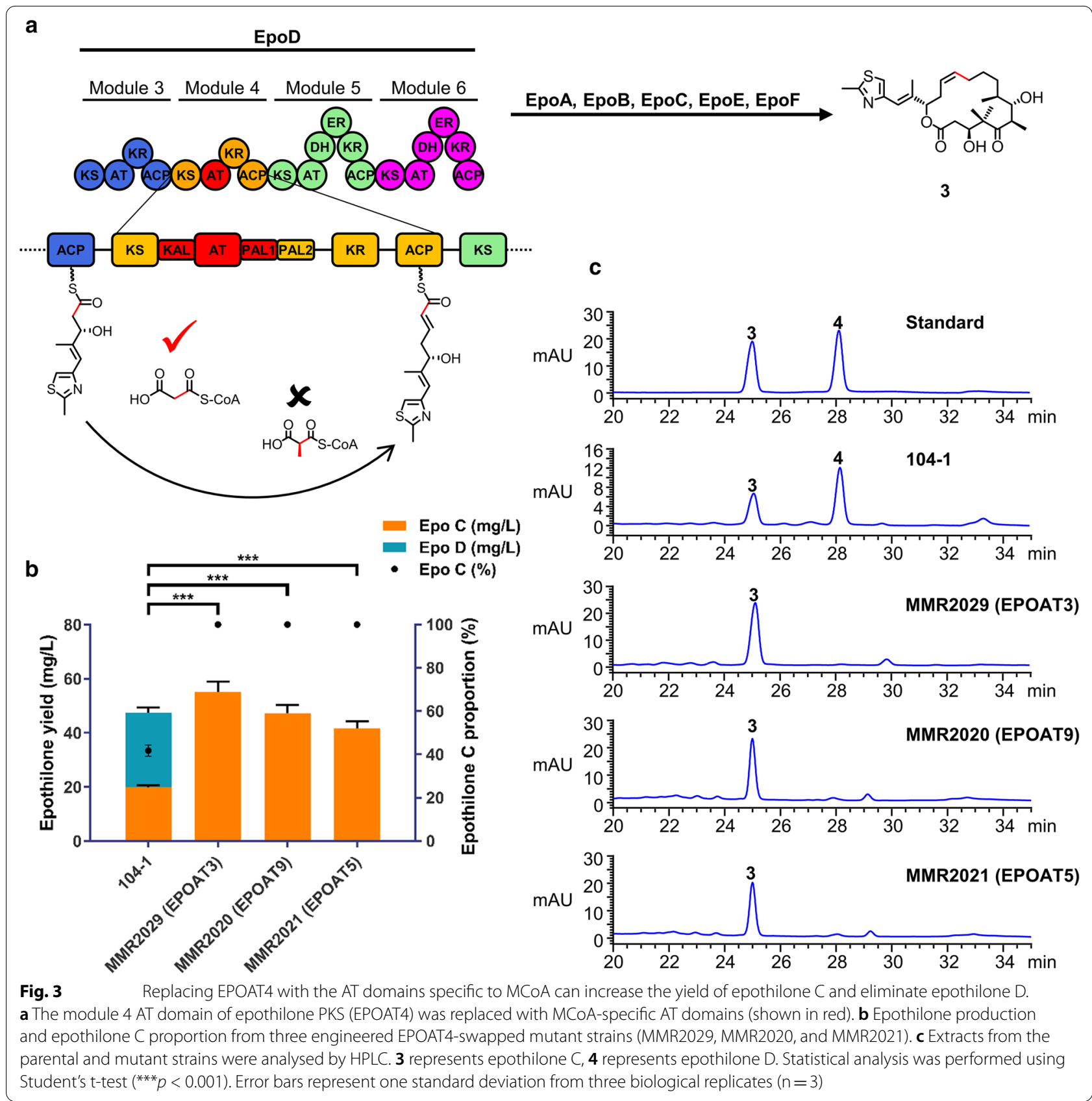

(VASH), and EPOAT4 (HASH) domains (Fig. 5c). It thus seemed reasonable that we could alter AT selectivity to cause predominant production of epothilone $\mathrm{D}$ by further mutating the hybrid motif to VASH or YASH (Fig. 5c). We mutated His308 from the hybrid HASH motif in MMR3035 (F310S) to the basic residue valine or tyrosine, resulting in MMR2041 (F310S-H308V) and MMR2042 (F310S-H308Y). As shown in Fig. 5a, b, the percentage of epothilone D increased from 36 to $43 \%$ in MMR2041. However, to our surprise, the percentage of epothilone $\mathrm{D}$ decreased to $25 \%$ in MMR2042. The total yields of MMR2035, MMR2041, and MMR2042 declined somewhat compared to MMR2029 (Additional file 1: Table S2). These results showed that His308 plays an auxiliary role in the substrate recognition of EPOAT4. 


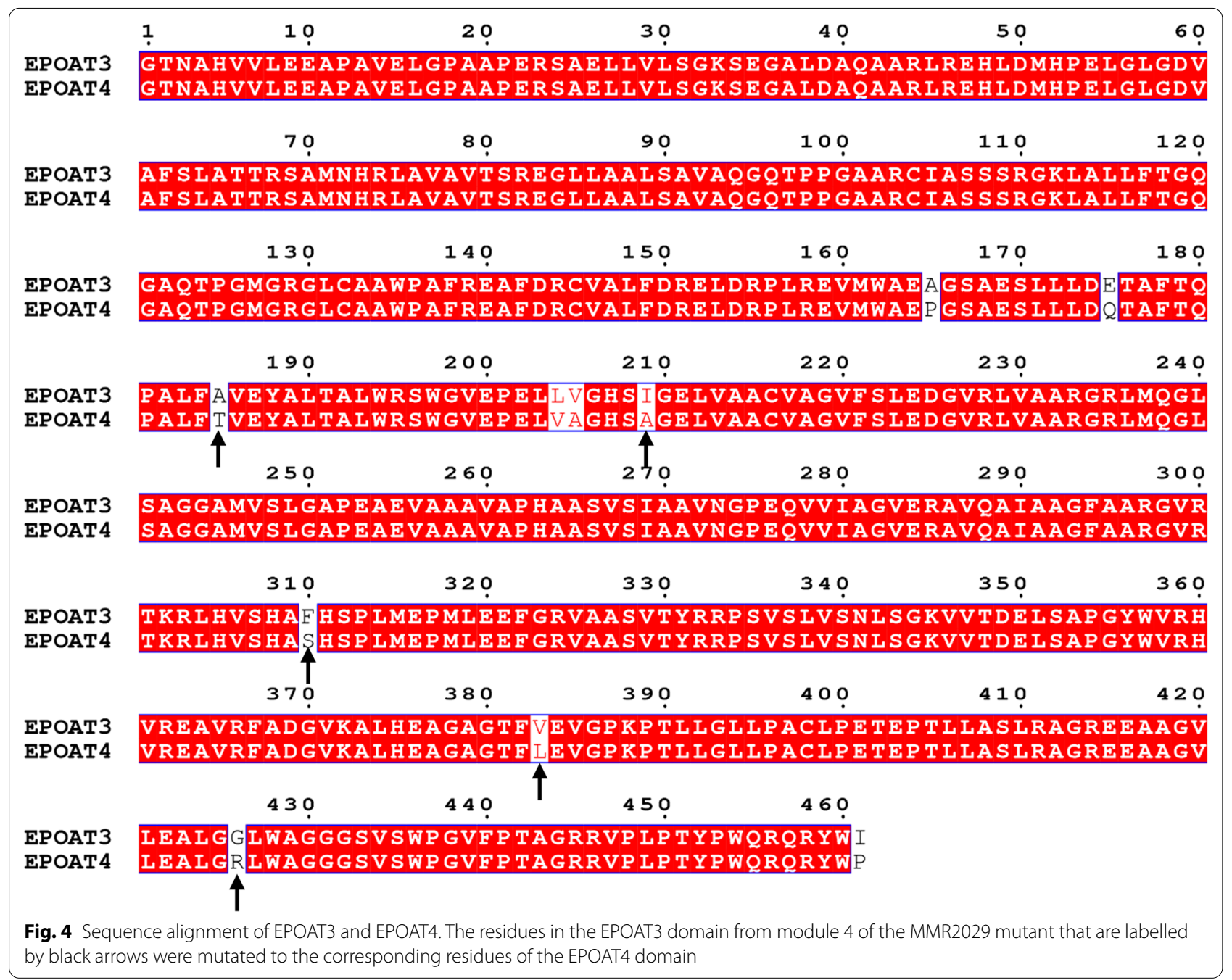

\section{Retention and linkage of $\Psi \mathrm{KR}$ with the KR domain are essential to obtain a productive epothilone PKS while deleting the $\mathrm{DH}$ and $\mathrm{ER}$ domains}

It can be deduced from the epothilone structures that the redundant EPODH9 and EPOER9 are not required. First, the DH9- KKR9-ER9 domains were deleted in 104-1 to generate MMR2037, and epothilone production was abolished entirely in this recombinant strain (Fig. 6a, b, c). We speculated that the inactivation of this epothilone PKS may be caused by the deletion of $\Psi K R$, since previous studies indicate that structural $\Psi K R$ could support the function of the catalytic KR, although $\Psi K R$ has lost the ability to bind NADPH due to the extensive truncation of its core [11,37]. Thus, we assumed that deleting DH9 and ER9 without affecting the structural integrity and activity of KR/YKR was a worthwhile approach to generating a productive epothilone PKS. In the followup experiments, we deleted the ER9 domain and DH9ER9 didomains but retained the YKR9 domain between
DH9 and ER9 to generate two strains, MMR2019 and MMR2038 (Fig. 6d). There were no differences in the epothilone composition compared to that of 104-1, and a small increase in total yield was detected in MMR2019 (50.6 mg/l) and MMR2038 (53.7 mg/l) (Fig. 6c; Additional file 1: Table S2). We successfully simplified the complete modifying region to a partial modifying region, and we reduced the size of the epothilone gene cluster by $1.9 \mathrm{~kb}$ through this method.

The efficient processivity of the modified epothilone PKS might be attributed to the use of optimal deletion sites. Amino acid sequence alignments between different modules were carried out (Additional file 1: Figure S8-S9), and when the ER9 domain was deleted in these two mutants, we retained an appropriate linker to interconnect the $\Psi$ KR9 and KR9 domains (Additional file 1: Figure S8). This modified $\Psi$ KR9-KR9-linker was the same length as the natural $\Psi \mathrm{KR}-\mathrm{KR}$-linker in modules that harbour a partial modifying region ( $\Psi K R-K R)$ 


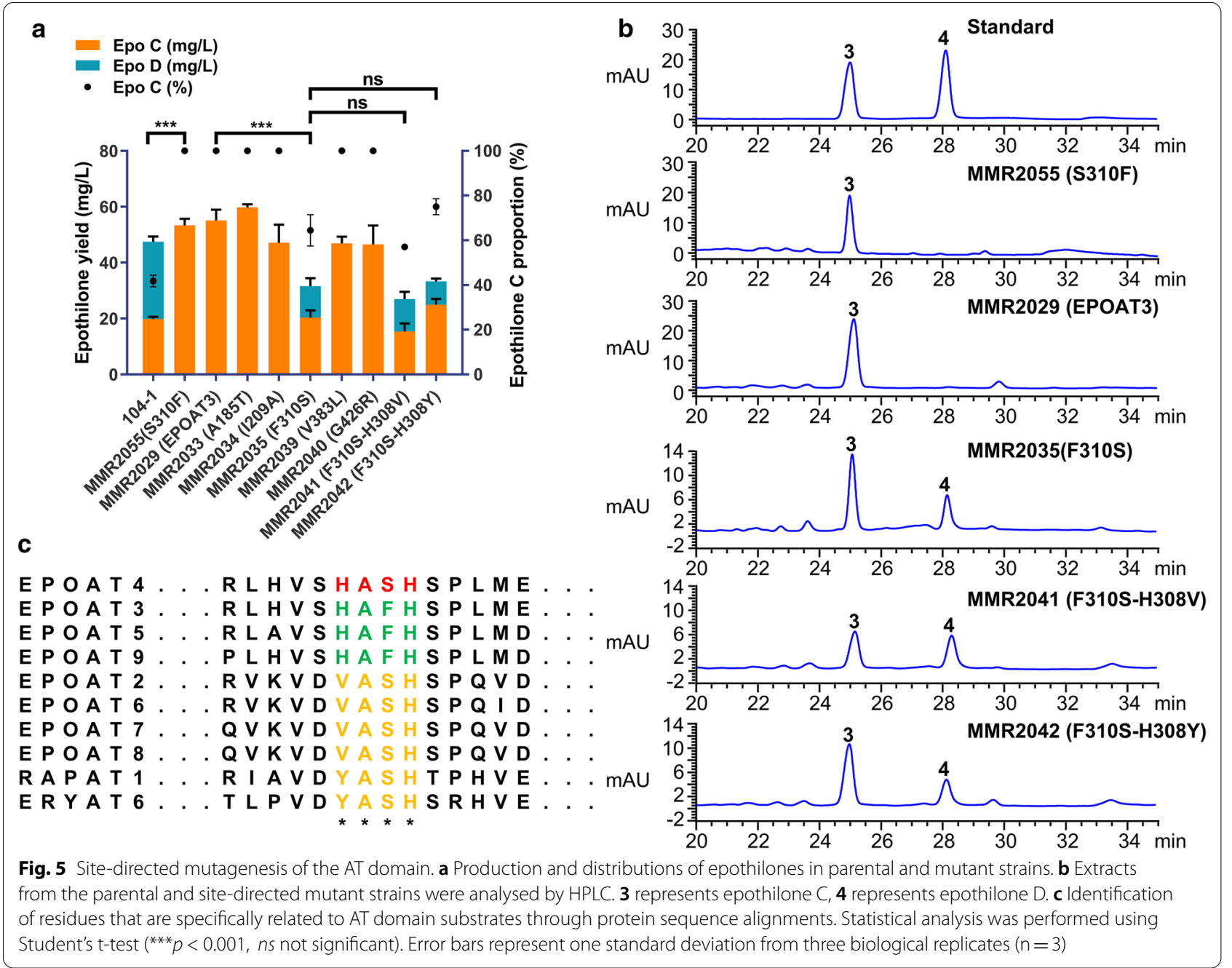

(Additional file 1: Figure S8). To investigate the importance and flexibility of $\Psi$ KR-KR-linker, $\Psi$ KR9-KR9linker was deleted in MMR2038 to give MMR2053 and was replaced by $\sim 25$-residue YKR3-KR3-linker from module 3 of the epothilone PKS (EPOM3) to generate MMR2054 (Fig. 6e). Predictably, the epothilone production was entirely abolished in MMR2053 (Fig. 6c), illustrating the removal of $\Psi$ KR9-KR9-linker destroyed the catalytic activity of the epothilone PKS. The model structures also showed that the $\sim 25$-residue $\Psi$ KR9KR9-linker plays a vital role in maintaining the catalytic activity of the KR9 domain (Additional file 1: Figure S1b). Interestingly, the epothilone PKS in MMR2054 still has catalytic activity, and the total epothilone yields in MMR2054 were $41.8 \mathrm{mg} / \mathrm{l}$ (Fig. 6c). This consequence indicated although the $\Psi$ KR-KR-linker is indispensable, it is flexible and could be replaced by other linkers between $\Psi K R$ and KR.

\section{Discussion}

In this study, we employed three PKS engineering strategies to produce singular metabolites, and the product distributions were altered. Compared with exchanging the whole module, domain-swap and site-directed mutagenesis methods are more effective methods. Furthermore, we successfully simplified EPOM9 by deleting inactive domains and fused $\Psi$ KR9 with the KR9 domain without decreasing the productivity of the epothilone PKS.

The activity levels of our module engineered systems were drastically diminished even though we retained appropriate covalent linkers to connect heterologous modules. We speculate that this result is attributable to the impaired specific interactions between upstream $\mathrm{ACP}\left(\mathrm{ACP}_{\mathrm{n}-1}\right)$ and downstream KS $\left(\mathrm{KS}_{\mathrm{n}}\right)$ domains during chain translocation [7, 38, 39]. Future attempts to design productive module-exchange PKSs must pay attention to optimize the $\mathrm{ACP}_{\mathrm{n}-1}-\mathrm{KS}_{\mathrm{n}}$ interaction, as 


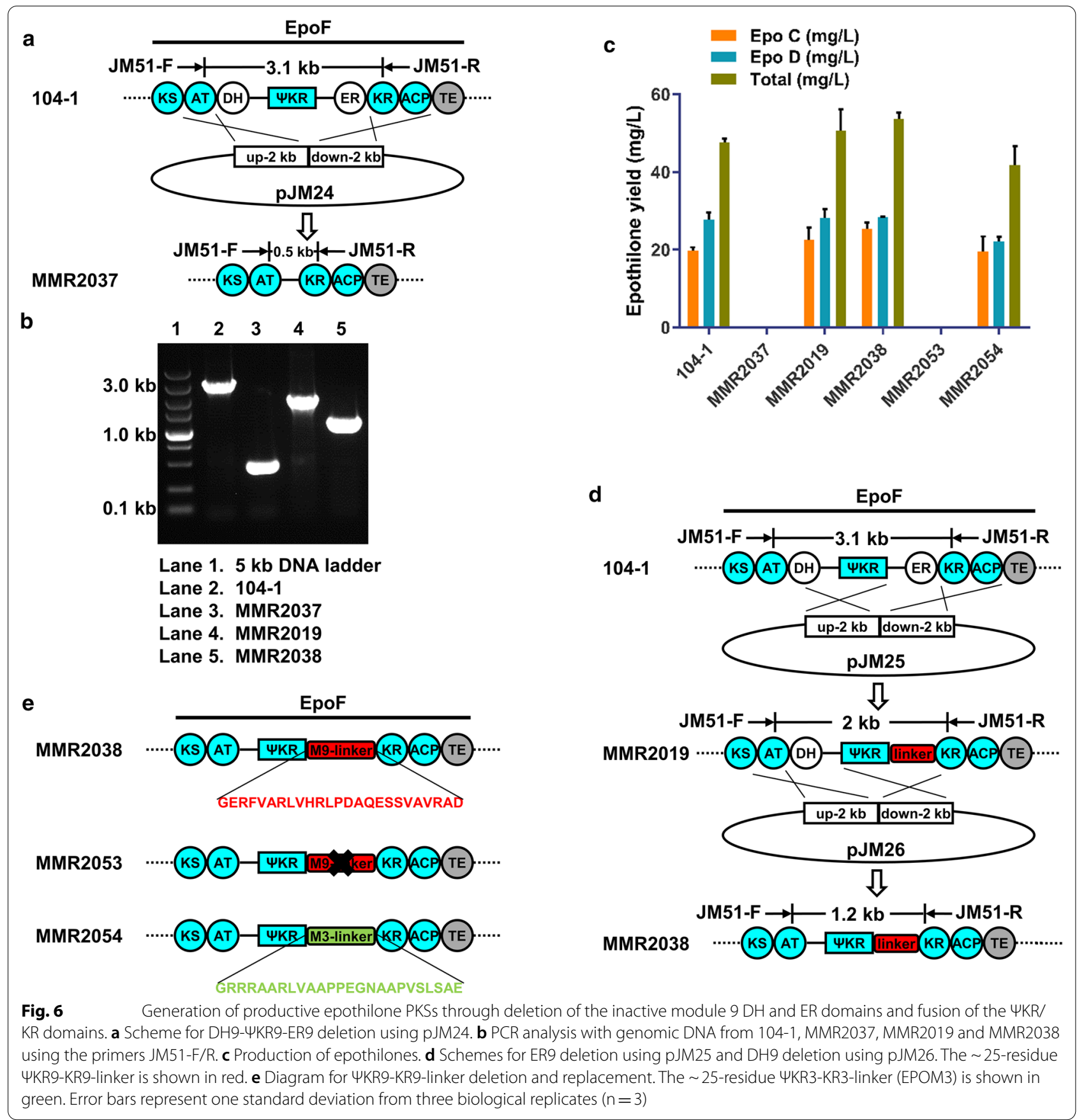

it has been suggested that mutation of the residue in $\mathrm{ACP}_{n-1}$, which was predicted to affect the $\mathrm{ACP}_{\mathrm{n}-1}-\mathrm{KS}_{\mathrm{n}}$ recognition, led to increased turnover [38].

In addition to exchanging the module, AT swapping and site-directed mutagenesis of AT are commonly employed strategies. Based on identification of the highly conserved AT-swap boundaries, hybrid AT-swap PKSs were constructed to produce over $1 \mathrm{~g} / \mathrm{l}$ short-chain ketone in vivo by Yuzawa et al. [40]. This AT-swap strategy has also been successfully used in this work. However, the generation of productive AT-swap PKSs cannot be guaranteed by employing optimized AT boundaries because much lower yields of epothilones were detected in the EPOAT4-swap strains MMR2018 (RAPAT1) and MMR2012 (ERYAT6). We speculated that this result was due to the disruption of AT-ACP interactions within one module, as previous studies indicate that certain residues of ACP are responsible for cognate AT recognition during chain elongation 
$[39,41,42]$. The successful AT swaps in this study may be attributed to the high sequence similarity between the EPOAT4 domain and other EPOAT domains derived from the same PKS as EPOAT4; it is possible that they recognize EPOACP4 through proper AT-ACP interactions.

Site-directed mutagenesis of AT has often led to diminished turnover rates of PKSs [22, 43]; in this work, the epothilone yields of mutants that harbour mutations F310S and $\mathrm{H} 308 \mathrm{~V} / \mathrm{Y}$ declined to a small degree compared to the starting strain. Mutation H308V had a better effect than $\mathrm{H} 308 \mathrm{Y}$ on the MMCoA specificity of AT and resulted in an improved ratio of epothilone D. However, mutating motif HAFH to YASH/VASH alone is not enough to reverse the substrate specificity of AT domains completely. Other complementary mutations that do not destroy the catalytic activity of the complete PKS are needed. For example, using saturation mutagenesis, Sundermann et al. found the mutation Q198L also resulted in the MCoA recognition of DEBS AT6 in addition the mutations in YASH motif [44].

The failure of the deletion of the DH9-YKR9-ER9 region in terms of activity suggested that although $\Psi K R$ is a noncatalytic pseudo-KR domain, retention of the $\Psi$ KR domain helps generate productive PKSs. The importance of $\Psi$ KR in supporting the integrity of the catalytic KR in PKSs and mammalian fatty acid synthase (mFAS) has also been described in previous studies [11, 32, 37, 45]. We successfully fused the $\Psi$ KR9 domain with the KR9 domain by an 25-residue linker in EPOM9 and maintained good catalytic activity in the KR9 domain, which is convenient for future KR9 domain engineering. Interestingly, we also found that the $\Psi$ KR-KR-linker is indispensable, but it may only play a role in connecting $\Psi K R$ and KR structurally and could be replaced by a linker of equal length from other modules.

\section{Conclusions}

In conclusion, functional hybrid epothilone PKSs were generated, and product distributions were altered by swapping the EPOAT4 domain and by site-directed mutation of the conserved motif in AT. Furthermore, we simplified module 9 of epothilone PKS by the deletion of inactive domains without decreasing the production and proved the importance and the sequence flexibility of $\Psi$ KR-KR-linker. These results enhance our understanding of PKS domains and provide new insights into PKS engineering.

\section{Methods}

\section{Chemicals, strains and culture conditions}

All bacterial strains used in this study are listed in Table 1. The primers used in this study are shown in Additional file 1: Table S4 and were synthesized by Genewiz (United States). DNA sequencing was performed by Tsingke
(China). The In-Fusion Cloning Kit was purchased from Novoprotein (China). DNA polymerases and a sitedirected mutagenesis kit were purchased from Vazyme (China). Restriction endonucleases were purchased from Thermo Fisher Scientific (United States). The $S$. brevitalea DSM 7029 strains were cultivated at $30{ }^{\circ} \mathrm{C}$ in CYMG broth or on CYMG agar [33]. Sucrose (3\%) was added when the $s a c B$ gene was introduced for negative selection [46]. The Escherichia coli DH10B strains were grown at $37^{\circ} \mathrm{C}$ in Luria-Bertani (LB) broth or on LB agar. Antibiotics were used where required at the following final concentrations: ampicillin, $100 \mu \mathrm{g} / \mathrm{ml}$; spectinomycin, $50 \mu \mathrm{g} / \mathrm{ml}$; apramycin, $50 \mu \mathrm{g} / \mathrm{ml}$; kanamycin, $50 \mu \mathrm{g} / \mathrm{ml}$; and hygromycin B, $150 \mu \mathrm{g} / \mathrm{ml}$ for E. coli and $100 \mu \mathrm{g} / \mathrm{ml}$ for S. brevitalea DSM 7029.

\section{Plasmid and strain construction}

The plasmids used in this study are shown in Additional file 1: Table S5. We discuss the method of construction of EPOAT4 replacement mutants as an example. To change the EPOAT4 domain, the recombinant plasmids pJM6pJM14 were constructed. Briefly, using the genomic DNA of $104-1$ as a template, the $2.0-\mathrm{kb}$ upstream arm and 1.9-kb downstream arm were amplified using primers JM01-F/R and JM02-F/R and added to PJM1 using InFusion cloning to generate pJM5. The epoAT2, epoAT6, epoAT7, ероAT8, ероAT3, ероAT5, and epoAT9 genes were cloned from the genome of $S$. brevitalea 104-1 by PCR. The rapAT1 and eryAT6 genes were amplified from the genomes of Streptomyces hygroscopicus and Saccharopolyspora erythraea, respectively. These fragments were ligated into pJM5 individually using In-Fusion cloning to form the recombinant plasmid pJM6-pJM14. After verification by restriction digestion analysis and sequencing, all recombinant plasmids were transformed into the parental strain S. brevitalea 104-1. After growing at $30{ }^{\circ} \mathrm{C}$ for 2.5 days, recombinant single-crossover colonies were selected on plates containing apramycin. We confirmed the integrated mutants by PCR and transferred the colonies into $2 \mathrm{ml}$ of antibiotic-free CYMG broth at $30{ }^{\circ} \mathrm{C}$. To force these mutants to undergo a second round of recombination, in the next three days, we transferred $10 \mu \mathrm{l}$ of the culture into $2 \mathrm{ml}$ of antibioticfree fresh CYMG broth three times, and 1-2\% sucrose was added in each step. Then, we replicated the colonies grown on CYMG agar plates without antibiotics on CYMG agar plates with apramycin. Colonies that grew only on antibiotic-free CYMG agar plates were screened for being double-crossover mutants. The expected upAT4swap and AT4swap-down fragments were obtained from a double crossover by PCR (Additional file 1: Figure S10a, b), and DNA sequencing further confirmed that EPOAT4 in the epothilone PKS was successfully 
Table 1 Strains used in this study

\begin{tabular}{|c|c|c|}
\hline Strains & Relevant characteristics & Source \\
\hline \multicolumn{3}{|l|}{ Escherichia coli } \\
\hline $\mathrm{DH} 10 \mathrm{~B}$ & Host strain for cloning & Invitrogen \\
\hline Saccharopolyspora erythraea & Erythromycin-producing strain & Lab stock \\
\hline Streptomyces hygroscopicus & Rapamycin-producing strain & Lab stock \\
\hline \multicolumn{3}{|l|}{ Schlegelella brevitalea } \\
\hline DSM 7029 & Wild type & Lab stock \\
\hline $104-1$ & Epothilone producing strain & Lab stock \\
\hline MMR2048 & Mutant strain of 104-1 with EPOAT4 replaced by EPOAT2 & This study \\
\hline MMR2049 & Mutant strain of 104-1 with EPOAT4 replaced by EPOAT6 & This study \\
\hline MMR2017 & Mutant strain of 104-1 with EPOAT4 replaced by EPOAT7 & This study \\
\hline MMR2016 & Mutant strain of 104-1 with EPOAT4 replaced by EPOAT8 & This study \\
\hline MMR2018 & Mutant strain of 104-1 with EPOAT4 replaced by RAPAT1 & This study \\
\hline MMR2012 & Mutant strain of 104-1 with EPOAT4 replaced by ERYAT6 & This study \\
\hline MMR2029 & Mutant strain of 104-1 with EPOAT4 replaced by EPOAT3 & This study \\
\hline MMR2021 & Mutant strain of 104-1 with EPOAT4 replaced by EPOAT5 & This study \\
\hline MMR2020 & Mutant strain of 104-1 with EPOAT4 replaced by EPOAT9 & This study \\
\hline MMR2032 & Mutant strain of MMR2029 with EPOAT3 in EPOM4 replaced by aadA & This study \\
\hline MMR2033 & Mutant strain of MMR2032 with aadA replaced by EPOAT3 (A185T) & This study \\
\hline MMR2034 & Mutant strain of MMR2032 with aadA replaced by EPOAT3 (I209A) & This study \\
\hline MMR2035 & Mutant strain of MMR2032 with aadA replaced by EPOAT3 (F310S) & This study \\
\hline MMR2039 & Mutant strain of MMR2032 with aadA replaced by EPOAT3 (V383L) & This study \\
\hline MMR2040 & Mutant strain of MMR2032 with aadA replaced by EPOAT3 (G426R) & This study \\
\hline MMR2041 & Mutant strain of MMR2032 with aadA replaced by EPOAT3 (F310S-H308V) & This study \\
\hline MMR2042 & Mutant strain of MMR2032 with aadA replaced by EPOAT3 (F310S-H308Y) & This study \\
\hline MMR2055 & Mutant strain of 104-1 with EPOAT4 (S310F) & This study \\
\hline MMR2037 & Mutant strain of 104-1 with EPODH9-4KR9-ER9 region deleted & This study \\
\hline MMR2019 & Mutant strain of 104-1 with EPOER9 deleted & This study \\
\hline MMR2038 & Mutant strain of MMR2019 with EPODH9 deleted & This study \\
\hline MMR2053 & Mutant strain of MMR2038 with $\Psi$ KR9-KR9-linker deleted & This study \\
\hline MMR2054 & $\begin{array}{l}\text { Mutant strain of MMR2038 with } \Psi \text { KR9-KR9-linker (EPOM9) replaced by } \Psi \text { KR3-KR3-linker } \\
\text { (EPOM3) }\end{array}$ & This study \\
\hline MMR2044 & Mutant strain of 104-1 with EPOM4 replaced by EPOM7 & This study \\
\hline MMR2024 & Mutant strain of 104-1 with EPOM4 replaced by ERYM6 & This study \\
\hline MMR2027 & Mutant strain of 104-1 with EPOM4 replaced by RAPM4 & This study \\
\hline MMR2026 & Mutant strain of 104-1 with EPOM4 replaced by RAPM10 & This study \\
\hline
\end{tabular}

replaced with non-native AT domains. The obtained double-crossover mutant strains were named MMR2048 (EPOAT2), MMR2049 (EPOAT6), MMR2017 (EPOAT7), MMR2016 (EPOAT8), MMR2018 (RAPAT1), MMR2012 (ERYAT6), MMR2029 (EPOAT3), MMR2021 (EPOAT5) and MMR2020 (EPOAT9).

The same method was used to construct EPOM4 replacement mutants, AT site-directed mutagenesis mutants, inactive domain deletion mutants and YKR9KR9-linker deletion and replacement mutants. Plasmids pJM29-pJM32 were constructed from pJM3, and the resulting EPOM4 replacement mutants were MMR2044
(EPOM7), MMR2024 (ERYM6), MMR2027 (RAPM4), and MMR2026 (RAPM10) (Additional file 1: Figure S11). The epothilone-negative strain MMR2032 was obtained to analyse the impact of mutations by insertional inactivation via homologous recombination, where the gene encoding EPOAT3 in EPOM4 of MMR2029 was replaced by an $\operatorname{add} A$ fragment encoding spectinomycin resistance. pJM15 was constructed from pJM4 to give MMR2032. We introduced mutation sites by site-directed mutagenesis using primers (Additional file 1: Table S4) and constructed pJM16-pJM23 from pJM5. The obtained site-directed mutagenesis strains MMR2033 (A185T), 
MMR2034 (I209A), MMR2035 (F310S), MMR2039 (V383L), MMR2040 (G426R), MMR2041 (F310SH308V), MMR2042 (F310S-H308Y) and MMR2055 (S310F) were assessed by PCR, and the resulting PCR products were sequenced (Additional file 1: Figure S12). For the deletion of inactive domains, we constructed plasmid pJM24 from pJM1, pJM25 from pJM3, and pJM26 from pJM2. The resulting mutants MMR2037, MMR2019, and MMR2038 were amplified by PCR using the primers JM51-F/R to verify removal (Fig. 6b; Additional file 1: Table S4). For the deletion and replacement of IKR9-KR9-linker, we constructed plasmid pJM27 from pJM1, pJM28 from pJM27. The resulting mutants MMR2053 and MMR2054 were assessed by PCR and the resulting PCR products were sequenced (Additional file 1: Figure S13).

\section{Fermentation, extraction and HPLC analysis of epothilone production}

A single colony was inoculated into $2 \mathrm{ml}$ of CYMG broth supplemented with the appropriate antibiotic and then incubated at $30^{\circ} \mathrm{C}$ and $220 \mathrm{rpm}$ for $24 \mathrm{~h}$. Then, a $250-\mathrm{ml}$ conical flask harbouring $30 \mathrm{ml}$ of CYMG broth and $300 \mu \mathrm{l}$ of overnight culture was incubated with shaking for $24 \mathrm{~h}$. Flask-scale fermentations were performed at $30{ }^{\circ} \mathrm{C}$ for 6 days in a 2-l shake flask with a 500-ml working volume. The fermentation medium $(500 \mathrm{ml})$ in the $2-1$ shake flask was inoculated with $25 \mathrm{ml}$ of preculture. The fermentation medium and extraction methods were described in our previous work [34]. We filtered the eluate by using $0.22-\mu \mathrm{m}$ organic filter membranes and diluted the extract by adding $100 \mu \mathrm{l}$ of extract to $900 \mu \mathrm{l}$ of ethyl acetate. The supernatant was analysed by HPLC using an Ultimate XB-C18 column $(4.6 \times 250 \mathrm{~mm}, 5 \mu \mathrm{m}$, Welch, Shanghai, China), and its absorbance was measured at $249 \mathrm{~nm}$ on a 1260 Infinity instrument (Agilent Technologies, United States) after centrifugation at 12,500 rpm for $15 \mathrm{~min}$. The flow rate was $1.0 \mathrm{ml} / \mathrm{min}$, and gradient elution was performed using mobile phase solvent $\mathrm{C}$ (water) and solvent $\mathrm{D}$ (methanol). The gradient conditions were as follows: 0-5 min, hold at $65 \% \mathrm{D} ; 5-15 \mathrm{~min}$, linear increase from 65 to $75 \% \mathrm{D} ; 15-25 \mathrm{~min}$, linear increase from 75 to $80 \%$ $\mathrm{D}$; and $25-40 \mathrm{~min}$, hold at $80 \%$. The injection volume was $10 \mu \mathrm{l}$.

\section{LC-MS analysis of epothilone production}

LC-MS analysis of epothilone production was conducted using the method described in our previous study [33]. The mass values were $m / z=478.4$ and 290.2 for epothilone C, 492.4 and 304.2 for epothilone D. A dilution series of epothilone standards was created to generate a concentration standard curve for quantification (Additional file 1: Figure S14). All samples were diluted to proper concentrations within the standard curve before detection.

\section{Multiple sequence alignment and structural modelling}

The amino acid sequences were aligned using ClustalW (https://www.genome.jp/tools-bin/clustalw), and the alignments were produced using ESPript 3.0 (http://espri pt.ibcp.fr/ESPript/cgi-bin/ESPript.cgi ).

Phyre2 (http://www.sbg.bio.ic.ac.uk/phyre2/html/page. cgi?id=index) was used to generate all tertiary structure models [47]. Structure alignment of the two models was conducted by PyMOL 2.3.2.

\section{Abbreviations}

PKs: Polyketides; NRPs: Nonribosomal peptides; PKSs: Polyketide synthases; NRPSs: Nonribosomal peptide synthetases; MCoA: Malonyl-CoA; MMCoA: Methylmalonyl-CoA; KS: Ketosynthase; AT: Acyltransferase; ACP: Acyl carrier protein; UKR/KR: Ketoreductase; DH: Dehydratase; ER: Enoylreductase; TE: Thioesterase; KAL: KS-AT linker; PAL: Post-AT linker.

\section{Supplementary Information}

The online version contains supplementary material available at https://doi. org/10.1186/s12934-021-01578-3.

Additional file 1: Table S1. Engineered module-swap fusion sites of hybrid epothilone PKSs. Table S2. Yields of epothilones in mutants of Schlegelella brevitalea DSM 7029. Table S3. Alignment of nine amino acids that differ in EPOAT3 and EPOAT4. Table S4. Primers used for the construction of plasmids and verification of mutant strains. Table S5. Plasmids used in this study. Figure $\mathbf{S 1}$. Structural models of reducing domains in wild-type and mutant EPOM9s. Figure S2. Engineering the epothilone PKS by whole-module swapping. Figure S3. Extracted ion chromatogram (EIC) of LC-MS analyses of epothilone production in EPOM4-swap mutants. Figure S4. Multiple sequence alignment of AT domains used in this study. Figure S5. Extracted ion chromatogram (EIC) of LC-MS analyses of epothilone production in some AT-swap mutants to further confirm the production of epothilones. Figure S6. Extracted ion chromatogram (EIC) of LC-MS analyses of epothilone production in AT-swap mutants MMR2012 and MMR2018. Figure S7. HPLC analysis of the extracts from the parental and site-directed mutant strains. Figure S8. Junctions for ER domain deletion constructs. Figure S9. Junctions for DH domain deletion constructs. Figure S10. PCR analysis for the confirmation of AT-swap mutants. Figure S11. PCR analysis for the confirmation of module-exchange mutants. Figure S12. DNA sequences of the PCR products of site-directed mutants. Figure S13. DNA sequences of the PCR products of KKR9-KR9-linker deletion mutant (MMR2053) and replacement mutant (MMR2054). Figure S14. Standard curves calculated based on the peak areas of epothilone standards of different concentrations.

\section{Acknowledgements}

Not applicable.

\section{Authors' contributions}

$X D, G Z, H W$ and $J L$ designed the research; HW, JL, QY and $Y S$ conducted the biological experiments; HW, JL, LL, GC, YL, XB, YZ, GZ and XD analysed the data; HW wrote and $X D$ revised the manuscript. All authors read and approved the final manuscript.

\section{Funding}

This work was financially supported by grants from the National Key R\&D Program of China (2020YFA0908300 and 2016YFA0500600), the National Natural Science Foundation of China (31970049), and the Science and Technology Research Program of Shanghai (19DZ2282100). 


\section{Availability of data and materials}

All data generated or analysed during this study are included in this published article and its supplementary information files.

\section{Declarations}

\section{Ethics approval and consent to participate}

Not applicable.

\section{Consent for publication}

Not applicable.

\section{Competing interests}

The authors declare that they have no competing interests.

\section{Author details}

${ }^{1}$ Collaborative Innovation Center for Genetics and Development, State Key Laboratory of Genetic Engineering, Shanghai Engineering Research Center of Industrial Microorganisms, Department of Microbiology, School of Life Sciences, Fudan University, Shanghai 200438, People's Republic of China. ${ }^{2}$ The State Key Laboratory of Molecular Engineering of Polymers, Department of Macromolecular Science, Fudan University, Shanghai 200433, People's Republic of China. ${ }^{3}$ State Key Laboratory of Microbial Technology, School of Life Sciences, Shandong University-Helmholtz Institute of Biotechnology, Shandong University, Qingdao, Shandong, People's Republic of China. ${ }^{4}$ CAS Key Laboratory of Synthetic Biology, Institute of Plant Physiology and Ecology, Shanghai Institutes for Biological Sciences, Chinese Academy of Sciences, Shanghai 200032, People's Republic of China.

Received: 4 January 2021 Accepted: 7 April 2021

Published online: 21 April 2021

\section{References}

1. Walsh CT, Fischbach MA. Natural products version 2.0: connecting genes to molecules. J Am Chem Soc. 2010;132(8):2469-93.

2. Wang H, Fewer DP, Holm L, Rouhiainen L, Sivonen K. Atlas of nonribosomal peptide and polyketide biosynthetic pathways reveals common occurrence of nonmodular enzymes. Proc Natl Acad Sci U S A. 2014;111(25):9259-64.

3. Hertweck $C$. The biosynthetic logic of polyketide diversity. Angew Chem Int Ed Engl. 2009;48(26):4688-716.

4. Marahiel MA. Working outside the protein-synthesis rules: insights into non-ribosomal peptide synthesis. J Pept Sci. 2009;15(12):799-807.

5. Weissman KJ. The structural biology of biosynthetic megaenzymes. Nat Chem Biol. 2015;11(9):660-70.

6. Fischbach MA, Walsh CT. Assembly-line enzymology for polyketide and nonribosomal Peptide antibiotics: logic, machinery, and mechanisms. Chem Rev. 2006;106(8):3468-96.

7. Robbins T, Liu YC, Cane DE, Khosla C. Structure and mechanism of assembly line polyketide synthases. Curr Opin Struct Biol. 2016;41:10-8.

8. Keatinge-Clay AT. The structures of type I polyketide synthases. Nat Prod Rep. 2012;29(10):1050-73

9. Williams GJ. Engineering polyketide synthases and nonribosomal peptide synthetases. Curr Opin Struct Biol. 2013;23(4):603-12.

10. Miyanaga A, Kudo F, Eguchi T. Protein-protein interactions in polyketide synthase-nonribosomal peptide synthetase hybrid assembly lines. Nat Prod Rep. 2018;35(11):1185-209.

11. Maier T, Leibundgut $M$, Ban $N$. The crystal structure of a mammalian fatty acid synthase. Science. 2008;321(5894):1315-22.

12. Koryakina I, Kasey C, McArthur JB, Lowell AN, Chemler JA, Li S, Hansen DA, Sherman DH, Williams GJ. Inversion of Extender Unit Selectivity in the Erythromycin Polyketide Synthase by Acyltransferase Domain Engineering. ACS Chem Biol. 2017;12(1):114-23.

13. Menzella HG, Reid R, Carney JR, Chandran SS, Reisinger SJ, Patel KG, Hopwood DA, Santi DV. Combinatorial polyketide biosynthesis by de novo design and rearrangement of modular polyketide synthase genes. Nat Biotechnol. 2005;23(9):1171-6.
14. Klaus M, Grininger M. Engineering strategies for rational polyketide synthase design. Nat Prod Rep. 2018;35(10):1070-81.

15. Barajas JF, Blake-Hedges JM, Bailey CB, Curran S, Keasling JD. Engineered polyketides: Synergy between protein and host level engineering. Synth Syst Biotechnol. 2017;2(3):147-66.

16. McDaniel R, Thamchaipenet A, Gustafsson C, Fu H, Betlach M, Ashley G. Multiple genetic modifications of the erythromycin polyketide synthase to produce a library of novel "unnatural" natural products. Proc Natl Acad Sci U S A. 1999:96(5):1846-51.

17. Mulzer J, Altmann K, Hoefle G, Mueller R, Prantz K. Epothilones - A fascinating family of microtubule stabilizing antitumor agents. CR Chim. 2008;11(11-12):1336-68.

18. Muller R. Biosynthesis and heterologous production of epothilones. Fortschr Chem Org Naturst. 2009:90:29-53.

19. Altaha R, Fojo T, Reed E, Abraham J. Epothilones: a novel class of non-taxane microtubule-stabilizing agents. Curr Pharm Des. 2002;8(19):1707-12.

20. Lechleider RJ, Kaminskas E, Jiang X, Aziz R, Bullock J, Kasliwal R, Harapanhalli R, Pope S, Sridhara R, Leighton J, et al. Ixabepilone in combination with capecitabine and as monotherapy for treatment of advanced breast cancer refractory to previous chemotherapies. Clin Cancer Res. 2008;14(14):4378-84

21. Xu B, Sun T, Zhang Q, Zhang P, Yuan Z, Jiang Z, Wang X, Cui S, Teng Y, $\mathrm{Hu}$ X, et al. Efficacy of utidelone plus capecitabine versus capecitabine for heavily pretreated, anthracycline- and taxane-refractory metastatic breast cancer: final analysis of overall survival in a phase III randomised controlled trial. Annals Oncol. 2021:32(2):218-28.

22. Petkovic H, Sandmann A, Challis IR, Hecht HJ, Silakowski B, Low L, Beeston N, Kuscer E, Garcia-Bernardo J, Leadlay PF, et al. Substrate specificity of the acyl transferase domains of EpoC from the epothilone polyketide synthase. Org Biomol Chem. 2008;6(3):500-6.

23. Yuzawa S, Deng K, Wang G, Baidoo EE, Northen TR, Adams PD, Katz L, Keasling JD. Comprehensive in vitro analysis of acyltransferase domain exchanges in modular polyketide synthases and Its application for short-chain ketone production. Acs Synth Biol. 2017;6(1):139-47.

24. Tang L, Chung L, Carney JR, Starks CM, Licari P, Katz L. Generation of new epothilones by genetic engineering of a polyketide synthase in Myxococcus xanthus. J Antibiot (Tokyo). 2005;58(3):178-84.

25. Tang L, Ward S, Chung L, Carney JR, Li Y, Reid R, Katz L. Elucidating the mechanism of cis double bond formation in epothilone biosynthesis. Am Chem Soc. 2004;126(1):46-7.

26. Oliynyk M, Brown M, Cortes J, Staunton J, Leadlay PF. A hybrid modular polyketide synthase obtained by domain swapping. Chem Biol. 1996:3(10):833-9.

27. Ruan X, Pereda A, Stassi DL, Zeidner D, Summers RG, Jackson M, Shivakumar A, Kakavas S, Staver MJ, Donadio S, et al. Acyltransferase domain substitutions in erythromycin polyketide synthase yield novel erythromycin derivatives. J Bacteriol. 1997;179(20):6416-25.

28. Lau J, Fu H, Cane DE, Khosla C. Dissecting the role of acyltransferase domains of modular polyketide synthases in the choice and stereochemical fate of extender units. Biochemistry-US. 1999-38(5):1643-51.

29. Stassi DL, Kakavas SJ, Reynolds KA, Gunawardana G, Swanson S, Zeidner D, Jackson M, Liu H, Buko A, Katz L. Ethyl-substituted erythromycin derivatives produced by directed metabolic engineering. P Natl Acad Sci USA. 1998:95(13):7305-9.

30. Schwecke T, Aparicio JF, Molnar I, Konig A, Khaw LE, Haydock SF, Oliynyk M, Caffrey P, Cortes J, Lester JB, et al. The biosynthetic gene cluster for the polyketide immunosuppressant rapamycin. Proc Natl Acad Sci U S A. 1995;92(17):7839-43.

31. Yu TW, Shen Y, Doi-Katayama Y, Tang L, Park C, Moore BS, Richard HC, Floss HG. Direct evidence that the rifamycin polyketide synthase assembles polyketide chains processively. Proc Natl Acad Sci U S A. 1999;96(16):9051-6.

32. Herbst DA, Jakob RP, Zahringer F, Maier T. Mycocerosic acid synthase exemplifies the architecture of reducing polyketide synthases. Nature. 2016:531(7595):533-7.

33. Yu Y, Wang H, Tang B, Liang J, Zhang L, Wang H, Bian X, Li YZ, Zhang Y, Zhao GP, et al. Reassembly of the Biosynthetic Gene Cluster Enables High Epothilone Yield in Engineered Schlegelella brevitalea. Acs Synth Biol. 2020;9(8):2009-22 
34. Liang J, Wang H, Bian X, Zhang Y, Zhao G, Ding X. Heterologous redox partners supporting the efficient catalysis of epothilone $B$ biosynthesis by EpoK in Schlegelella brevitalea. Microb Cell Fact. 2020;19(1):180.

35. Hardt IH, Steinmetz H, Gerth K, Sasse F, Reichenbach H, Hofle G. New natural epothilones from Sorangium cellulosum, strains So ce90/B2 and So ce90/D13: isolation, structure elucidation, and SAR studies. J Nat Prod. 2001;64(7):847-56.

36. Del VF, Petkovic H, Kendrew SG, Low L, Wilkinson B, Lill R, Cortes J, Rudd BA, Staunton J, Leadlay PF. Active-site residue, domain and module swaps in modular polyketide synthases. J Ind Microbiol Biotechnol. 2003;30(8):489-94.

37. Keatinge-Clay AT, Stroud RM. The structure of a ketoreductase determines the organization of the beta-carbon processing enzymes of modular polyketide synthases. Structure. 2006;14(4):737-48.

38. Klaus M, Ostrowski MP, Austerjost J, Robbins T, Lowry B, Cane DE, Khosla C. Protein-protein interactions, not substrate recognition, dominate the turnover of chimeric assembly line polyketide synthases. J Biol Chem. 2016;291(31):16404-15.

39. Kapur S, Lowry B, Yuzawa S, Kenthirapalan S, Chen AY, Cane DE, Khosla C. Reprogramming a module of the 6-deoxyerythronolide B synthase for iterative chain elongation. Proc Natl Acad Sci U S A. 2012;109(11):4110-5.

40. Yuzawa S, Mirsiaghi M, Jocic R, Fujii T, Masson F, Benites VT, Baidoo E, Sundstrom E, Tanjore D, Pray TR, et al. Short-chain ketone production by engineered polyketide synthases in Streptomyces albus. Nat Commun. 2018;9(1):4569.

41. Ye Z, Musiol EM, Weber T, Williams GJ. Reprogramming acyl carrier protein interactions of an Acyl-CoA promiscuous trans-acyltransferase. Chem Biol 2014, 21(5):636-646.
42. Miyanaga A, Iwasawa S, Shinohara Y, Kudo F, Eguchi T. Structure-based analysis of the molecular interactions between acyltransferase and acyl carrier protein in vicenistatin biosynthesis. Proc Natl Acad Sci U S A. 2016;113(7):1802-7.

43. Reeves CD, Murli S, Ashley GW, Piagentini M, Hutchinson CR, McDaniel R. Alteration of the substrate specificity of a modular polyketide synthase acyltransferase domain through site-specific mutations. Biochemistry-US. 2001;40(51):15464-70.

44. Sundermann U, Bravo-Rodriguez K, Klopries S, Kushnir S, Gomez H, Sanchez-Garcia E, Schulz F. Enzyme-directed mutasynthesis: a combined experimental and theoretical approach to substrate recognition of a polyketide synthase. Acs Chem Biol. 2013;8(2):443-50.

45. Rittner A, Paithankar KS, Drexler DJ, Himmler A, Grininger M. Probing the modularity of megasynthases by rational engineering of a fatty acid synthase Type I. Protein Sci. 2019;28(2):414-28.

46. Lei X, Fan Q, Huang T, Liu H, Zhao G, Ding X. Efficient circular gene knockout system for Burkholderiales strain DSM 7029 and Mycobacterium smegmatis mc2 155. Acta Biochim Biophys Sin (Shanghai). 2019;51(7):697-706.

47. Kelley LA, Mezulis S, Yates CM, Wass MN, Sternberg MJ. The Phyre2 web portal for protein modeling, prediction and analysis. Nat Protoc. 2015;10(6):845-58.

\section{Publisher's note}

Springer Nature remains neutral with regard to jurisdictional claims in published maps and institutional affiliations.
Ready to submit your research? Choose BMC and benefit from:

- fast, convenient online submission

- thorough peer review by experienced researchers in your field

- rapid publication on acceptance

- support for research data, including large and complex data types

- gold Open Access which fosters wider collaboration and increased citations

- maximum visibility for your research: over $100 \mathrm{M}$ website views per year

At BMC, research is always in progress.

Learn more biomedcentral.com/submissions 